\title{
The Effect of Beta on the Downstream Development of Unstable, Chaotic Baroclinic Waves
}

\author{
JOSEPH PEDLOSKY \\ Physical Oceanography Department, Woods Hole Oceanographic Institution, Woods Hole, Massachusetts
}

(Manuscript received 24 April 2019, in final form 8 July 2019)

\begin{abstract}
The weakly unstable, two-layer model of baroclinic instability is studied in a configuration in which the flow is perturbed at the inflow section of a channel by a slow and periodic perturbation. In a parameter regime where the governing equation would be the Lorenz equations for chaos if the development occurs only in time, the solution behavior becomes considerably more complex as a function of time and downstream coordinate. In the absence of the beta effect it has earlier been shown that the chaotic behavior along characteristics renders the solution nearly discontinuous in the slow downstream coordinate of the asymptotic model. The additional presence of the beta effect, although expunging the chaos for large enough values of the beta parameter, also provides an additional mechanism for abrupt spatial change.
\end{abstract}

\section{Introduction}

Starting with the work of Lorenz (1963) the manifestation of chaotic behavior in unstable baroclinic systems has usually been examined in the context of the development of the instability in time. Although the Lorenz equations were introduced as a truncated model of thermal convection they can be derived in a straightforward way in weakly nonlinear baroclinic flows without arbitrary truncation of a Fourier representation of the complete solution thus allowing more confident use in similar problems (Pedlosky and Frenzen 1980). More recently Pedlosky (2011, hereafter P11) examined the development of baroclinic unstable waves in space and time, as the disturbance moves downstream from an upstream source of perturbation energy such as might occur in currents like the separated Gulf Stream. That work showed how the Lorenz dynamics along characteristics could lead to abrupt spatial change in the amplitude of the developing disturbance. In the parameter regime that would be chaotic, if examined in the time domain alone, chaotic development along neighboring characteristics of the dynamics developing in time and downstream coordinate introduces this new and important feature to the dynamics. Neighboring characteristics with only slightly different initial data evolving according to the Lorenz model on each characteristic

\footnotetext{
Corresponding author: Joseph Pedlosky, jpedlosky@whoi.edu
}

will eventually have solutions that diverge by order one because of the exquisite sensitivity to initial conditions that is the nature of chaos. Solutions that diverge by order one on closely neighboring characteristics imply rapid change of amplitude in the downstream coordinate. This rapid change in behavior in the downstream coordinate has been called chaotic shocks (P11) and it is distinguishable from the more common shocks in fluid dynamics because the rapid change is not due to intersection of the systems characteristics but rather due to the chaotic development along parallel characteristics.

One of the simplifications in the analysis in P11 was the neglect of the beta effect. For narrow currents with large vertical shear the nondimensional parameter measuring the importance of beta in the quasigeostrophic potential vorticity equation is $\beta L^{2} / U_{s} \pi^{2}$, where $L$ is the width of the current, $U_{s}$ is the characteristic velocity of the vertical shear, and $\beta$ is the planetary vorticity gradient. For widths on the order of $100 \mathrm{~km}$ and velocities on the order of $1 \mathrm{~m} \mathrm{~s}^{-1}$ this parameter is on the order of $10^{-2}$. Although small, the nonlinear dynamics of the unstable wave is very sensitive to the beta effect as has been shown in an earlier work (Pedlosky 1981, hereafter P81). The beta effect introduces a term in the amplitude equations that tends to shield the unstable point at the origin of the solution phase plane from the solution trajectories and, as a consequence, for even small values of beta the solution asymptotes to a periodic solution whose amplitude is determined by one of the two points in the phase plane 
representing fixed amplitude solutions (aside from a linear frequency depending on beta). The phase of the oscillation amplitude is not determined by this quasi-steady solution. This implies that the possibility exists for the solution, developing in space and time, allows neighboring characteristics to carry amplitudes differing in sign so that rapid variations in the solution amplitude occur. That is, there is the possibility that solutions on neighboring characteristic may differ in sign even when the solution is no longer behaving chaotically and thus can introduce rapid, shock-like behavior in the downstream coordinate. The purpose of this paper is to investigate this possibility and, in fact, demonstrate the existence of such solutions so that "chaotic shocks" can occur even when the solution along characteristics is only briefly chaotic.

Section 2 of the paper derives the governing equations. Section 3 presents numerical examples of the hypothesized behavior and in the concluding section, section 4 , the implication of the results is discussed.

\section{Formulation}

We start with the two-layer model in a channel of width $L$ governed by the quasigeostrophic potential vorticity equations, namely, for $n=1,2$ :

$$
\begin{gathered}
\frac{\partial}{\partial t}\left[\nabla^{2} \psi_{n}+F(-1)^{n}\left(\psi_{1}-\psi_{2}\right)\right]+J\left(\psi_{n}, \nabla^{2} \psi_{n}\right. \\
\left.+F(-1)^{n}\left(\psi_{1}-\psi_{2}\right)+\beta y\right)=-r \nabla^{2} \psi_{n} .
\end{gathered}
$$

The equations are nondimensional. Lengths have been scaled by $L$, velocities by a characteristic velocity $U$ of the initial basic flow, and time by $L / U$. The layers are of equal depth $D$ so the rotational Froude number $F=f^{2} L^{2} / g^{\prime} D$, where $g^{\prime}$ is the reduced gravity. The nondimensional parameter $\beta=\beta_{\operatorname{dim}} L^{2} / U$ while the dissipation parameter $r=(\nu f / 2)^{1 / 2} L /(U D)$, where $f$ is the Coriolis parameter and $\nu$ is the kinematic viscosity. The symbol $J(a, b)$ is defined as $a_{x} b_{y}-a_{y} b_{x}$, where subscripts denote differentiation. The coordinate $x$ is in the downstream direction while $y$ measures distance across the stream.

It is convenient to write the equations in terms of the barotropic and baroclinic streamfunctions, $\psi_{B}=$ $(1 / 2)\left(\psi_{1}+\psi_{2}\right), \psi_{T}=\psi_{1}-\psi_{2}$, respectively. In the problem to be considered, the basic state consists of a uniform flow in each layer with a barotropic and baroclinic component so that the associated streamfunctions are

$$
\begin{aligned}
& \psi_{B}=-U_{B} y+\varphi_{B}(x, y, t), \\
& \psi_{T}=-U_{T} y+\varphi_{T}(x, y, t) .
\end{aligned}
$$

where the functions $\varphi_{B}, \varphi_{T}$ are the barotropic and baroclinic perturbation streamfunctions. They satisfy the nonlinear equations

$$
\begin{aligned}
& \frac{\partial \nabla^{2} \varphi_{B}}{\partial t}+U_{B} \frac{\partial \nabla^{2} \varphi_{B}}{\partial x}+\frac{U_{T}}{4} \frac{\partial \nabla^{2} \varphi_{T}}{\partial x} \\
& \quad+J\left(\varphi_{B}, \nabla^{2} \varphi_{B}\right)+\frac{1}{4} J\left(\varphi_{T}, \nabla^{2} \varphi_{T}\right)+\beta \frac{\partial \varphi_{B}}{\partial x}=-r \nabla^{2} \varphi_{T} \\
& \frac{\partial}{\partial t}\left(\nabla^{2} \varphi_{T}-2 F \varphi_{T}\right)+U_{B} \frac{\partial\left(\nabla^{2} \varphi_{T}-2 F \varphi_{T}\right)}{\partial x}+2 F U_{T} \frac{\partial \varphi_{B}}{\partial x} \\
& \quad+J\left(\varphi_{T}, \nabla^{2} \varphi_{B}\right)+J\left(\varphi_{B}, \nabla^{2} \varphi_{T}-2 F \varphi_{T}\right) \\
& \quad+\beta \frac{\partial \varphi_{T}}{\partial x}=-r \nabla^{2} \varphi_{T} .
\end{aligned}
$$

The beta parameter will be considered a small (but important) perturbation to the dynamics. The critical curve for instability is therefore given at lowest order as a relation between $F_{c}$, the critical value of $F$, and the wavenumber with components $k$ and $l$ in the $x$ and $y$ directions, $\left(K^{2}=k^{2}+l^{2}\right)$, and is independent of $\beta$, that is,

$$
F_{c}=K^{2} / 2+\frac{r K^{2} / k}{2 U_{T}} .
$$

For small values of $r$ the minimum occurs at very long wavelengths and this informs our choice of scaling for the problem's variables. We make the following assumptions:

(i) The basic flow is only slightly supercritical with respect to $F$ so that

$$
F=F_{c}+\Delta, \quad \Delta \ll 1,
$$

(ii) The beta parameter and dissipation are also small, $\beta=O\left(\Delta^{1 / 2}\right), r=O(\Delta)$.

(iii) The solution will be a function of "fast" and "slow" space and time variables. The fast variables correspond to the advection of the marginally stable wave by the barotropic mean flow as suggested by the linear problem. The slow variables describe the slow evolution of the slightly unstable wave. With these presumptions in mind we introduce a new fast space coordinate $\xi$, a new slow space coordinate $X$, a new fast time coordinate $\tau$, and a slow time coordinate $T$, each defined by

$$
\begin{array}{ll}
\xi=\Delta^{1 / 2} x, & X=\Delta x, \\
\tau=\Delta^{1 / 2} t, & T=\Delta t,
\end{array}
$$

and we will consider the perturbation streamfunction to be functions of $\xi, X, \tau$, and $T$ such that, for example,

$$
\frac{\partial \varphi}{\partial x} \Rightarrow \Delta^{1 / 2} \frac{\partial \varphi}{\partial \xi}+\Delta \frac{\partial \varphi}{\partial X}
$$




$$
\begin{aligned}
& \frac{\partial \varphi}{\partial t} \Rightarrow \Delta^{1 / 2} \frac{\partial \varphi}{\partial \tau}+\Delta \frac{\partial \varphi}{\partial T}, \\
& \text { for } \varphi=\varphi_{B} \text { or } \varphi_{T} .
\end{aligned}
$$

So, for example. the baroclinic perturbation potential vorticity becomes

$q_{T}=\left[\frac{\partial^{2}}{\partial y^{2}}+\Delta \frac{\partial^{2}}{\partial \xi^{2}}+2 \Delta^{3 / 2} \frac{\partial^{2}}{\partial \xi \partial X}+\Delta^{2} \frac{\partial^{2}}{\partial X^{2}}-2\left(F_{c}+\Delta\right)\right] \varphi_{T}$,

with similar representations throughout (2.3a) and (2.3b).

The perturbations streamfunctions will be expanded in an asymptotic series in the small amplitude, $\varepsilon=O\left(\Delta^{1 / 2}\right)$, of the perturbation

$$
\begin{aligned}
& \varphi_{B}=\varepsilon\left(\varphi_{B}^{(0)}+\varepsilon \varphi_{B}^{(1)}+\varepsilon^{2} \varphi_{B}^{(2)}+\cdots\right), \\
& \varphi_{T}=\varepsilon\left(\varphi_{T}^{(0)}+\varepsilon \varphi_{T}^{(1)}+\varepsilon^{2} \varphi_{T}^{(2)}+\cdots\right) .
\end{aligned}
$$

Inserting these transformations into (2.3a) and (2.3b) leads in a straightforward way to a set of lengthy equations, and only the pertinent results, easily checked, will be presented in this paper.

At the lowest order in $\varepsilon$ we obtain the results consistent with linear theory,

$$
\begin{aligned}
& \varphi_{B}^{(0)}=A e^{i k(\xi-c \tau)} \sin \pi y+* \\
& \varphi_{T}^{(0)}=0, \quad c=U_{B}, \quad F_{c}=l^{2} / 2, \quad l=\pi,
\end{aligned}
$$

where $*$ denotes the complex conjugate of the preceding expression.

At the next order in $\varepsilon$ we obtain an expression for the baroclinic perturbation,

$$
\begin{aligned}
\varphi_{T}^{(1)}= & \frac{4}{k U_{T}}\left[i\left(\frac{\partial}{\partial T}+U_{B} \frac{\partial}{\partial X}\right) A+\frac{i r}{\Delta} A+\frac{\beta k}{\Delta^{1 / 2} l^{2}} A\right] \\
& \times e^{i \vartheta} \sin \pi y+*+\Phi_{T}(X, y, T),
\end{aligned}
$$

where the final term in (2.10) is the baroclinic correction to the mean flow and is a function of only the slow $x$ and slow time variables as well as $y$. Note that the beta term enters as a term proportional to the frequency of the long Rossby wave.

With the above expressions it is now possible to calculate the nonlinear interaction terms, that is, the Jacobians at next order and obtain as the governing equation for $\Phi_{T}$

$$
\begin{aligned}
\left(\frac{\partial}{\partial T}\right. & \left.+U_{B} \frac{\partial}{\partial X}\right)\left(\frac{\partial^{2} \Phi_{T}}{\partial y^{2}}-2 F_{c} \Phi_{T}\right)+\frac{r}{\Delta} \frac{\partial^{2} \Phi_{T}}{\partial y^{2}} \\
= & -\frac{\varepsilon}{\Delta^{1 / 2}} \frac{4\left(2 F_{c} l\right)}{U_{T}}\left[\left(\frac{\partial}{\partial T}+U_{B} \frac{\partial}{\partial X}\right)|A|^{2}\right. \\
& \left.+\frac{2 r}{\Delta^{1 / 2}}|A|^{2}\right] \sin 2 l y .
\end{aligned}
$$

As long as $\varepsilon \ll \Delta$, which is a basic presumption since the dynamics is quasigeostrophic, the geostrophic velocity in the $y$ direction produced by the mean flow correction must vanish at $y=0,1$ which in turn implies that a solution to (2.11) proportional to $\sin 2 l y, l=\pi$, is appropriate. Hence a solution of the form $\Phi_{T}=P(X, T) \sin 2 l y$ leads to the governing equation for $P$,

$$
\begin{aligned}
& \left(\frac{\partial}{\partial T}+U_{B} \frac{\partial}{\partial X}\right) P+\frac{4}{5} \frac{r}{\Delta} P \\
& \quad=-\frac{\varepsilon}{\Delta} \frac{4}{5}\left[\left(\frac{\partial}{\partial T}+U_{B} \frac{\partial}{\partial X}\right)|A|^{2}+\frac{2 r}{\Delta}|A|^{2}\right] .
\end{aligned}
$$

Now that the equation for the baroclinic mean flow correction is determined, the governing equation for the evolution of the wave amplitude $A$ is determined as a solvability condition at $O\left(\Delta^{3 / 2}\right)$. After considerable but straightforward algebra we obtain

$$
\begin{gathered}
\left(\frac{\partial}{\partial T}+U_{B} \frac{\partial}{\partial X}\right)^{2} A+\frac{3}{2}\left(\frac{r}{\Delta}-i \frac{\beta k}{\Delta^{1 / 2} l^{2}}\right)\left(\frac{\partial}{\partial T}+U_{B} \frac{\partial}{\partial X}\right) A \\
-\sigma^{2} A-\frac{\varepsilon}{\Delta^{1 / 2}} \frac{k U_{T}}{8 l^{2}} A P=0
\end{gathered}
$$

$\sigma^{2}=\frac{\left(2-k^{2}\right) k^{2} U_{T}^{2}}{8 l^{2}}-\frac{r^{2}}{2 \Delta}+\frac{i r}{\Delta} \frac{\beta k}{\Delta^{1 / 2} l^{2}}+\frac{\beta^{2} k^{2}}{2 \Delta l^{4}}$.

A rescaling of the variables,

$T^{\prime}=\sigma T, \quad X^{\prime}=\frac{\sigma X}{U_{T}}, \quad A=A_{o} A^{\prime}, \quad P=P_{o} P^{\prime}$,

$b=\frac{\beta k}{8 \sigma \Delta^{1 / 2} l^{2}}$,

where

$P_{o}=\frac{\sigma^{2} \Delta^{1 / 2}}{\varepsilon k^{2} l U_{t}}, \quad A_{o}^{2}=\frac{5}{4} P_{o}, \quad \gamma=\frac{r}{\Delta \sigma}$,

allows the governing equations to be rewritten (after dropping primes from the new dependent variables) as

$$
\begin{aligned}
& \left(\frac{\partial}{\partial T}+\frac{\partial}{\partial X}\right)^{2} A+\frac{3}{2}(\gamma+i b)\left(\frac{\partial}{\partial T}+\frac{\partial}{\partial X}\right) A \\
& -A(1+P)=0 \\
& \left(\frac{\partial}{\partial T}+\frac{\partial}{\partial X}\right) P+\frac{4}{5} \gamma P=-\left[\left(\frac{\partial}{\partial T}+\frac{\partial}{\partial X}\right)|A|^{2}+2 \gamma P\right] .
\end{aligned}
$$

As a final change of variables we let $P=-|A|^{2}+R$, yielding, 
(a)

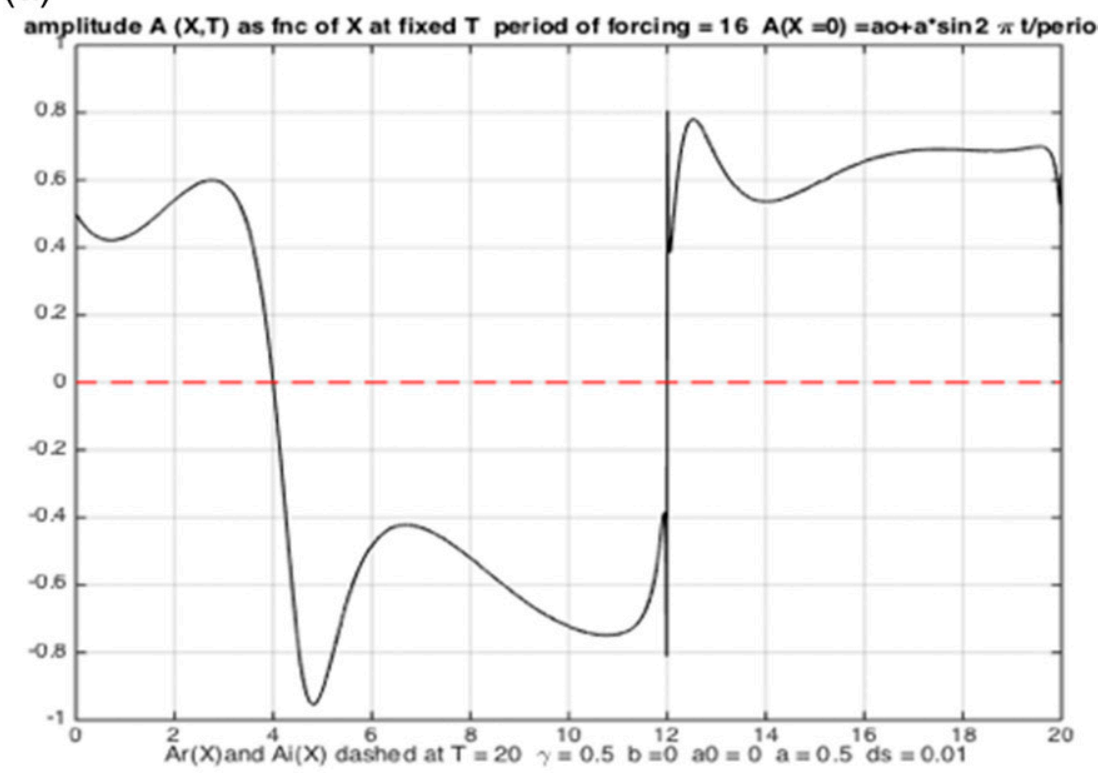

(b)

b) evolution of Ar and Ai (dashed) on neighboring char acteristics

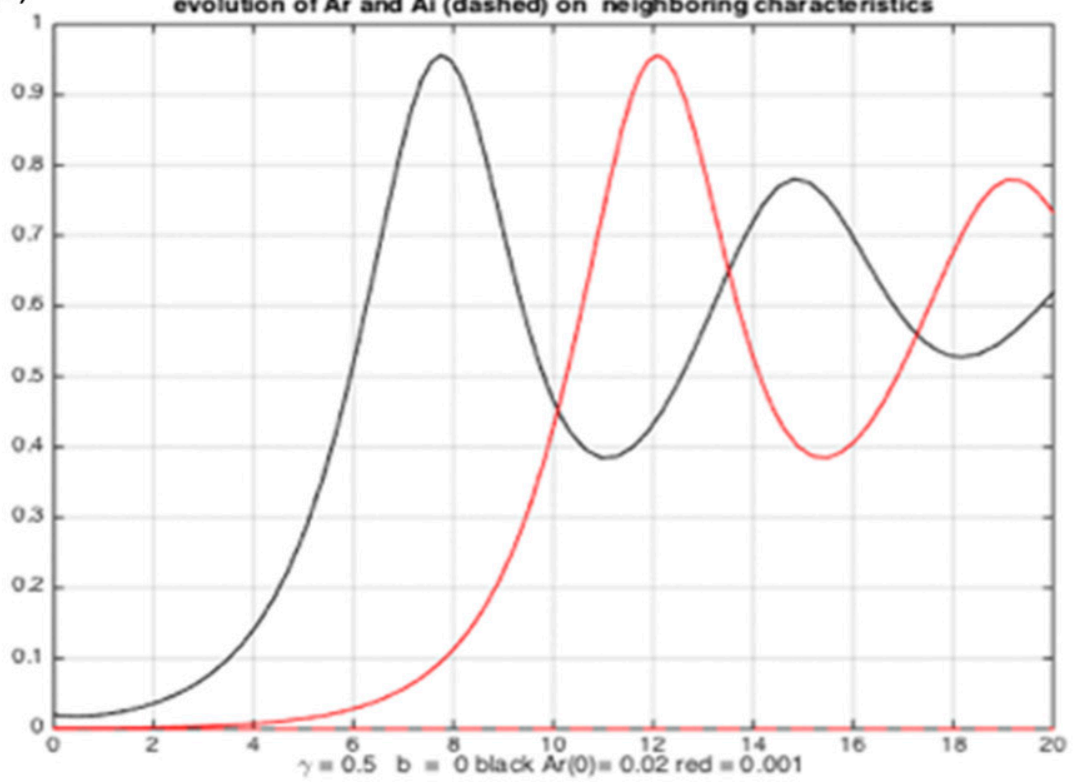

FIG. 1. (a) The solution as a function of $X$ for $A$ at $T=20, \gamma=0.5$, and $b=0$. With real boundary conditions at $X=0$ the imaginary part of $A$ remains zero. (b) The solution along two closely spaced characteristics. The chaotic nature of the solution leads to diverging values of $A$ rendering the solution rapidly varying in $X$.

$$
\begin{gathered}
\left(\frac{\partial}{\partial T}+\frac{\partial}{\partial X}\right)^{2} A+\frac{3}{2}(\gamma+i b)\left(\frac{\partial}{\partial T}+\frac{\partial}{\partial X}\right) A \\
-A+A\left(|A|^{2}+R\right)=0, \\
\left(\frac{\partial}{\partial T}+\frac{\partial}{\partial X}\right) P+\frac{4}{5} \gamma P=\frac{6}{5} \gamma|A|^{2},
\end{gathered}
$$

as our final evolution equations. The amplitude $A$ is complex, with real and imaginary parts, so the system of first-order partial differential equations given by (2.16) is fifth order. The characteristics of each of the five equations are the straight lines in the $X, T$ plane,

$$
T-X=T_{o},
$$


(a)

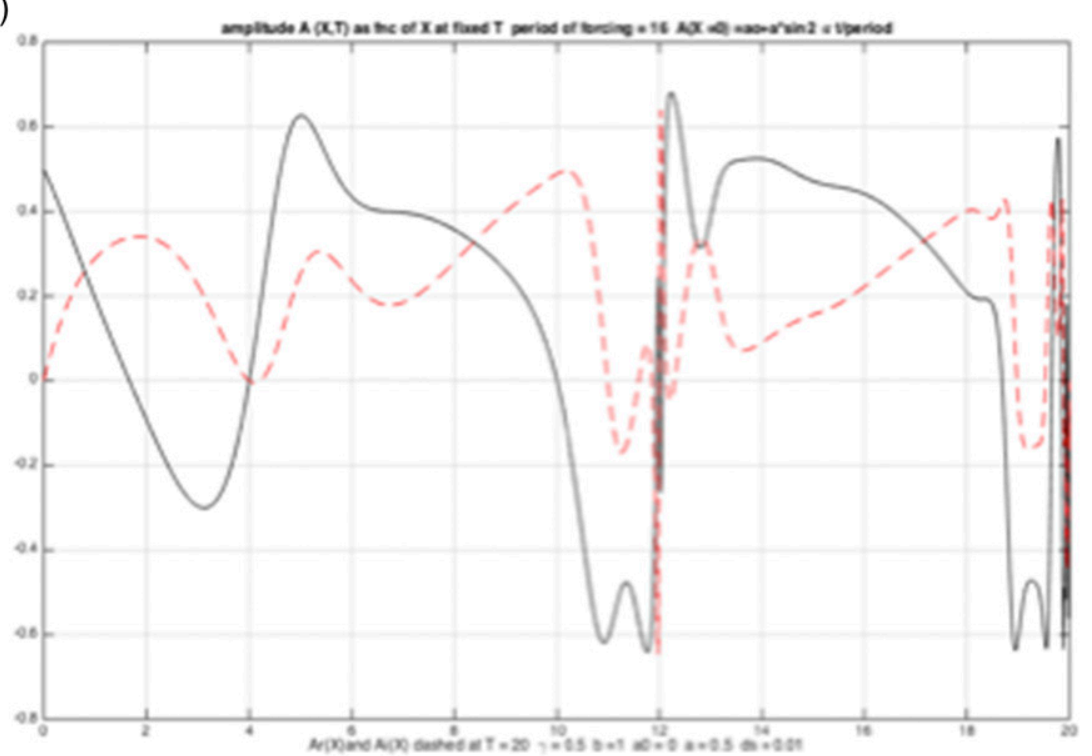

(b)

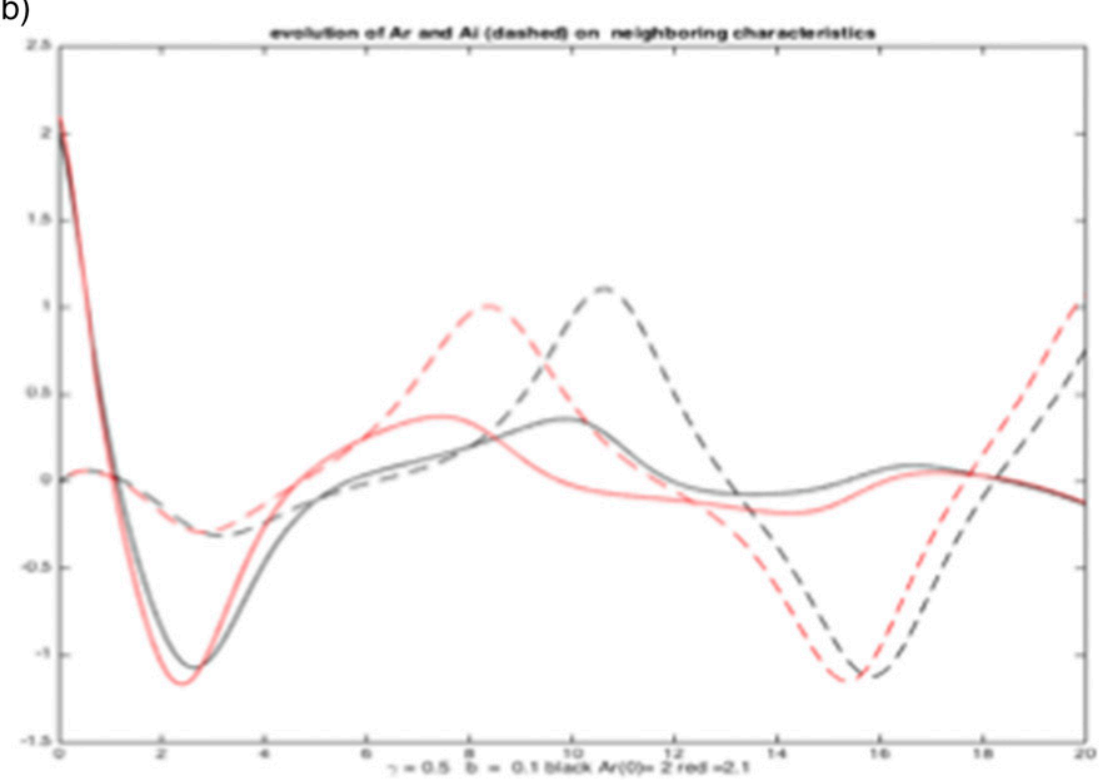

FIG. 2. As in Fig. 1, but now $b$ is 0.1 and sufficiently small so that the chaotic behavior is not suppressed along characteristics. The real and imaginary parts of $A$ are shown in (a), which both suffer rapid change in the slow variable $X$. The divergence of solutions on neighboring characteristics is shown again in (b).

where $T_{o}$ is the intersection of the characteristic with the $T$ axis at $X=0$. The variable $s$, representing distance in $X, T$ space along the characteristics renders (2.16a) and (2.16b) as a set of ordinary differential equations along the characteristics with the operator $\partial / \partial T+\partial / \partial X \Rightarrow d / d s$.

In the absence of the beta term, that is, for $b=0$, the resulting third-order system is equivalent to the Lorenz equations as shown in P81 and has chaotic solutions in $s$ for a certain range of $\gamma$.

\section{Results}

The system (2.16) is forced by the boundary condition at $X=0$ which we choose as

$$
A\left(0, T=T_{o}\right)=a \sin 2 \pi T / T_{\text {period }},
$$

where both $T_{\text {period }}$ and $a$ are given along with the parameters $\gamma, b$.

When $b=0$ we recover the results of $\mathrm{P} 11$, that is for sufficiently small $\gamma$ the Lorenz dynamics along the 
(a)

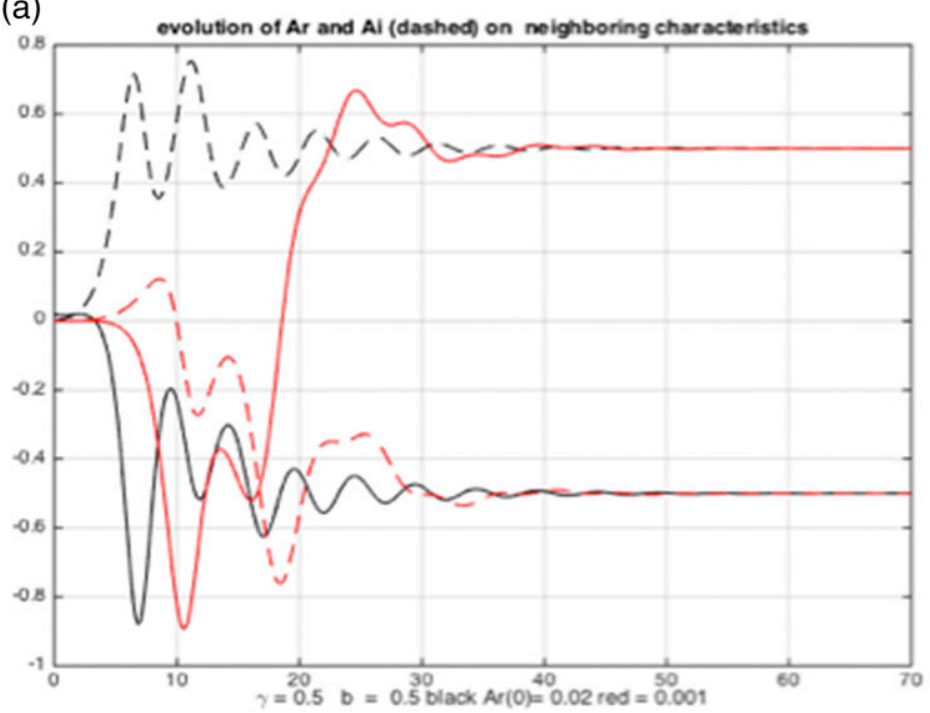

(b)

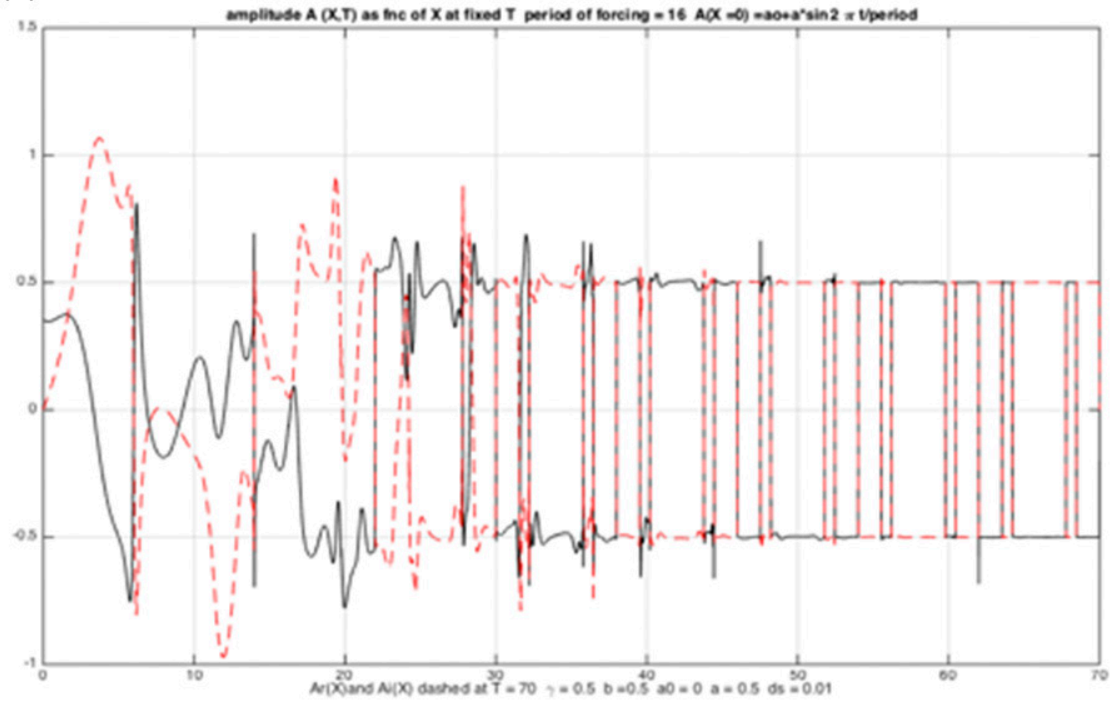

FIG. 3. (a) The solution along two closely spaced characteristics for $b=0.5$ for the same value of $\gamma$ as Fig. 1. (b) The sequence of shock-like changes in $X$ even though the chaos on characteristics is largely quenched.

characteristics of the partial differential equations of (2.16) yield chaotic solutions that diverge from slightly different initial conditions. For the problem of development in space and time this implies that neighboring characteristics with slightly different initial conditions from (2.18) will eventually diverge by $O(1)$ yielding values of $A$ at a given time that abruptly change with $X$. An example is shown in Fig. 1. In Fig. 1b the evolution along the characteristic curves is shown for slightly different initial data corresponding to two closely spaced characteristics. The divergence of the solutions, a standard feature of the Lorenz model implies extremely rapid change in $X$ for fixed $T$.
Figure 2 shows a similar behavior when $b$ is small (0.1) but nonzero. There is still sufficient divergence of the solutions along neighboring characteristics to lead to rapid change in $X$.

When $b$ is increased further (Fig. 3) to $b=0.5$, perhaps the most interesting behavior takes place. Panel a shows the evolution along two closely spaced characteristics. After a relatively brief period of chaotic behavior along the characteristics, the solution along each is captured by one of the two fixed points of the solution space. The fixed points both have the same value of $|A|^{2}$ but differ in phase: $A$ differs by a sign, that is, $A$ is positive on one characteristic and negative on the 


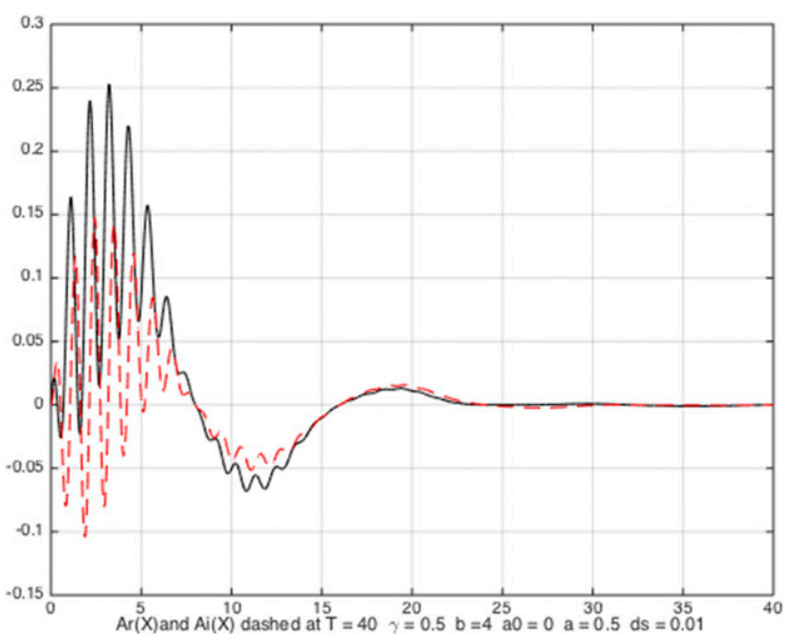

FIG. 4. For $b=4$, the chaotic behavior is absent and the solution in $X$ is smooth.

other. This implies that the amplitude itself will abruptly change in value in $X$. That wild behavior is exhibited in Fig. 3b. It means that even a brief period of chaotic behavior that puts the solution on a trajectory to be captured by a different fixed point has a violent manifestation in space that is rather unexpected.

Further increase in $b$ quenches the chaotic behavior completely as shown in Fig. 4. For $b=4$, the solution is smooth in $X$.

\section{Discussion}

The presence of the planetary beta effect has been earlier shown (P81) to have a strong effect on the chaotic behavior of weakly nonlinear, slightly unstable baroclinic instability. From a mathematical point of view the beta effect acts in the governing differential equations as a repulsive mechanism that keeps the solution trajectory from closely approaching the unstable point at the origin of the solution space that is the generator of the chaos. This has importance consequences for the model of the development of the instability as it grows and propagates in the downstream direction. With the presence of chaotic behavior along characteristics in the downstream and time slow coordinates, neighboring characteristics have solutions that diverge by order one in spite of their closeness and this leads to abrupt changes in the space variable of the system of equations. The introduction of a value of beta large enough expunges the chaos and smooths the solution in space. However, the presence of beta also can yield abrupt changes in the solutions dependence on space even when the solutions along the characteristics are chaotic for only a brief period of time and subsequently captured by one of the two fixed points differing only by a sign as shown in Fig. 3.

Of course, the abruptness of the solution behavior in space for the solutions of (2.16a) and (2.16b), while of interest for all systems governed by the Lorenz system of equations, really implies for the weakly nonlinear system in our problem the collapse of the separation between the slow behavior in time and the expected slow behavior in space. It is important to remember that for the parameters chosen the evolution in time is still slow and weak. The implication that the accompanying behavior in space may be qualitatively different requires further study of the original system without the asymptotic assumptions which are normally made, and usually so illuminating, but must be extended in future work.

\section{REFERENCES}

Lorenz, E. N., 1963: Deterministic non-periodic flow. J. Atmos. Sci., 20, 130-141, https://doi.org/10.1175/1520-0469(1963)020<0130: $\mathrm{DNF}>2.0 . \mathrm{CO} ; 2$

Pedlosky, J., 1981: The effect of $\beta$ on the chaotic behavior of unstable baroclinic waves. J. Atmos. Sci., 38, 717-731, https://doi.org/ 10.1175/1520-0469(1981)038<0717:TEOOTC $>2.0$.CO;2.

_ 2011: The nonlinear downstream development of baroclinic instability. J. Mar. Res., 69, 705-722, https://doi.org/ 10.1357/002224011799849363.

-, and C. Frenzen, 1980: Chaotic and periodic behavior of finite-amplitude baroclinic waves. J. Atmos. Sci., 37, 1177-1196, https://doi.org/10.1175/1520-0469(1980)037<1177: $\mathrm{CAPBOF}>2.0 . \mathrm{CO} ; 2$. 